\title{
Local Binary Patterns Applied to Breast Cancer Classification in Mammographies
}

\author{
Eanes Torres Pereira ${ }^{1}$ \\ Sidney Pimentel Eleutério ${ }^{2}$ \\ João Marques de Carvalho ${ }^{3} 4$
}

\begin{abstract}
Among all cancer types, breast cancer is the one with the second highest incidence rate for women. Mammography is the most used method for breast cancer detection, as it reveals abnormalities such as masses, calcifications, asymmetries and architectural distortions. In this paper, we propose a classification method for breast cancer that has been tested for six different cancer types: CALC, CIRC, SPIC, MISC, ARCH, ASYM. The proposed approach is composed of a SVM classifier trained with LBP features. The MIAS image database was used in the experiments and ROC curves were generated. To the best of our knowledge, our approach is the first to handle those six different cancer types using the same technique. One important result of the proposed approach is that it was tested over six different breast cancer types proving to be generic enough to obtain high classification results in all cases.
\end{abstract}

\section{Introduction}

Breast cancer is the second most common type of women cancer in the world [11] and a great cause of death, specially for women between 40 and 55 years of age [13]. In Brazil, the National Cancer Institute (INCA) estimated the occurrence of 52, 680 new cancer cases for the year 2012 [12]. In 2010, 12, 705 Brazilian women died due to breast cancer. One of the major reasons for those deaths is late cancer detection. When symptoms arise and are perceived it may be already too late for any treatment.

Mammography is one of the best methods for early detection of breast cancer signs [28]. It may reveal abnormalities such as masses, calcifications, asymmetries and architectural distortions [11]. Rangayyan et al. [25] affirm that the use of mammographic screening has effectively reduced breast cancer mortality rates by $30 \%$ to $70 \%$.

\footnotetext{
${ }^{1}$ Universidade Federal de Campina Grande, Departamento de Sistemas e Computação, Paraíba, Brasil \{eanes@computacao.ufcg.edu.br\}

${ }^{2}$ Universidade Estadual da Paraíba, Departamento de Computação, Paraíba, Brasil \{sidneyspe@gmail.com\}

${ }^{3}$ Universidade Federal de Campina Grande, Coordenação de Pós-Graduação em Informática, Paraíba, Brasil \{carvalho@dee.ufcg.edu.br\}

${ }^{4}$ Work partially supported by NUTES (Núcleo de Tecnologias Estratégicas em Saúde) http://nutes . uepb. edu.br
} 
A mammography is a detailed radiography of thorax, which allows a physician is able to detect anomalies even before perceiving the existence of nodes. Mammography inspection is the best isolated test to detect anomalies that may be a breast cancer early sign. Through its use it is possible to verify breast changes 2 years before either patient or physician are capable of perceiving it by tactile examination [26, p. 387]. Among the most frequent breast cancer signs early detectable by mammography is the occurrence of masses and micro-calcifications. However, mammography interpretation is not easy. The quality of a diagnostic obtained by mammography is affected by image quality as well as by the expertise of the radiologist.

Computer-aided diagnostic techniques (CAD) may offer a low cost alternative to aid the doctor on achieving a diagnostic. The purpose of those techniques is to reduce the occurrence of errors committed by the specialist. A CAD system works as a second reader, proposing suspicious mammographic areas for further examination. There are studies indicating the effectiveness of using CAD systems as a double reading method [6] [7] [8].

The double reading works as follows: 1) the physician performs the first mammography reading and marks suspicious regions, 2) the CAD system analyzes the image searching for signs of breast cancer, 3 ) the system outputs to the physician marked regions where cancer indications have been detected. The physician can then analyze the CAD system indications, to confirm or not the presence of cancer signs.

There are two types of CAD systems: computer-aided detection (CADe) and computer aided diagnosis (CADx). CADe systems aid in the process of detection and localization of regions with cancer signs. CADx systems aid in classifying the regions as, for example, benign or malignant. Generally, appearance-based methods are used for both types of CAD systems. When those methods are applied, regions of interest (ROI) are extracted and automatically classified as suspect $\times$ not-suspect or benign $\times$ malignant. The approach we propose in this paper aims to classify mammography image regions in one of the following categories: calcification, circumscribed masses, spiculated masses, ill-defined masses, architectural distortion, asymmetry, and normal.

The major contribution of the approach we propose is the use of texture features extracted with LBP (Local Binary Patterns) for classifying mammography images. Our approach is evaluated using ROC curves analysis. Considering that true positive rate (TPR) is the same as recall (fraction of retrieved relevant instances) [4], one may see from the results in Section 4 that for 4 of 6 categories of breast abnormalities the proposed approach achieved TPR results higher than $90 \%$. For the other 2 cases, the results were lower but near $90 \%$. These results are higher than those presented by other authors, for the same task but in some cases testing different data bases.

This paper is organized as follows. In Section 2 related works are presented. Our proposed approach is explained in Section 3 Section 4 presents experimental results. Finally, 
conclusions are summarized in Section 5

\section{Related Works}

In this section, we present a review of published work related to our proposal. The reviewed papers are grouped in two categories, those that use LBP as features for mammograms classification (Section 2.2) and those that use other techniques (Section 2.1)

\subsection{Non LBP approaches}

Lan et al.[13] proposed an approach for CAD systems that uses a hybrid classifier composed of logistic regression (LR) and k-nearest neighbor classifiers (KNN). They implemented a 2D segmentation method based on dynamic programming (DP) for extracting suspect masses in regions of interest. The authors used a multidimensional vector for describing 60 different features including gray levels, texture, shape, and edges; and used images from DDSM [5] (Digital Database for Screening Mammography) which is public available on web. Image resolution was fixed at $128 \times 128$ pixels and 800 regions of interest selected for training and testing classifiers. The approach obtained AUC (Area Under the Curve) of 0.86 . Independent test of the two classifiers, resulted in AUCs of 0.843 and 0.835 for logistic regression and k-nearest neighbors, respectively.

The performance of six different Gabor feature extraction methods was investigated by Hussain et al.[11] for the problem of mass classification in mammographies. The authors used transform techniques PCA and LDA to remove redundancy that may be caused by Gabor filter banks. For mass classification, a SELwSVM (Successive Enhancement Learning based weighted Support Vector Machine) classifier was employed. The images used for training and testing were obtained from 89 regions of interest acquired from the MIAS [17] (Mammographic Image Analysis Society) database. The metrics used for evaluating classifiers were area under the ROC curve (AUC) and cross-validation. Two binary classification problems sequentially were evaluated in sequence for each region of interest: mass versus normal and benign versus malignant. For each case, one classifier obtained maximum result: $100 \%$ of accuracy. However, it must be noted that the quantity of images used in the experiments was very small.

Nguyen et al. [18] proposed a method to detect tissue lesions and masses in mammographies using a Gray Level Co-occurrence Matrix (GLCM) and Haralick features extracted from regions of interest. Neural networks were applied as classifiers. For training and testing, 322 images from 161 patients were obtained from MIAS image database. The images were submitted to a preprocessing stage consisting of top-hat and bottom-hat operations for emphasizing peaks and maximizing contrast, respectively. The performance evaluation re- 
sult presented is 0.876 for the area under the ROC curve (AUC) which the authors argue is accurate enough.

Instead of simply train and test classifiers and generate a ROC curve for the results as was performed by Nguyen et al. [18], Zhang e Xie[28] used a 10-fold cross-validation procedure for base model selection in their proposed method for clustered micro-calcifications detection in mammograms. They applied a bagging procedure with cross-validation to generate base models for twin support vector machines (TWSVM). The classifiers were trained using feature based histogram, multi-scale histograms, Chebyshev transform histogram, and Zernike moments. The best classifier obtained using bagging with TWSVM achieved an AUC of 0.9627 . It is not possible to directly compare this result with Nguyen et al. [18] results, because they used different image databases. While Nguyen et al. [18] used images from MIAS database, Zhang e Xie [28] used images from DDSM image database.

Anitha and Peter[1] presented a method for classifying mammographic image regions as either mass or non-mass using wavelet features. The results are compared with results obtained by Nguyen et al. [18]. However, there are some differences between the approaches. Anitha and Peter[1] employed support vector machines (SVM) in the classification process instead of neural networks and 44 images from MIAS database for evaluating their approach. Those images were preprocessed such that the regions used for training and testing have resolution of $32 \times 32$ pixels. An important step of this approach is the segmentation of candidate regions involving opening operations, erosion, dilation, morphological reconstruction, connected component labeling, and computation of mass lesion areas. A true positive rate of $95.56 \%$ was obtained, higher than those of all other 5 approaches compared in the paper.

\subsection{LBP related papers}

The utilization of LBP in mammograms classification is very diverse, it has been applied for segmentation [15] and for classification [3, 15, 16]. In this subsection, we discuss some works in these two modes of application.

Liu et al. [15], proposed a method for mass segmentation and detection using an rulebased algorithm called MCL (multiple concentric layers) and NBAC (narrow band regionbased active contour). CLBP (Complete Local Binary Pattern) are extracted from ROIs of segmented suspicious regions. The selected regions are classified using SVM. The system obtained 1.36 FPsI(average number of false positive detections per image) at the sensitivity $76.8 \%$. The images used in the experiments were obtained from the craniocaudal views of the DDSM dataset, totaling 231 images (125 for training SVM and optimize MCL parameters, and 106 for testing). The main criticism to this method is that MCL is empirically optimized and the rules obtained for an image dataset may not be applied to a different image dataset.

Lladó et al. [16] proposed an approach for classifying ROIs as either breast cancer 
masses or normal parenchyma by grouping LBP features in order to better characterize texture micro-patterns not loosing spatial structure of masses. The authors used in the experiments 1792 ROIs (256 depicted as true mass and 1536 as normal tissue) extracted from the DDSM database (Digital Database for Screening Mammography). The approach applied for texture extraction is well known in face image processing [23, 24] and consists of extracting features both globally and locally. Global LBP histograms are obtained by computing the histogram of the overall LBP matrix. Local LBP histograms are obtained by dividing the LBP matrix in regions and for each region a histogram is computed. The authors proposed the use of a concatenation of those histograms to describe the image ROI texture. The results were also evaluated in terms of ratio between quantity of masses ROI and quantity of normal tissue ROIs, the results and ratios were $0.94 \pm 0.02(1 / 1), 0.94 \pm 0.02(1 / 2)$, and $0.91 \pm 0.04(1 / 3)$. One important aspect of locally extracted LBP is that it is not rotation invariant, although this is not mentioned by the authors. The influence of the shape of the masses on the performance of the method was not investigated.

Choi et al. [3] proposed the classification of mammograms in breast masses or normal tissue, using multi-resolution LBP classified by SVM-RFE [9](Support Vector Machine Recursive Feature Elimination). The main contributions of this work are the extraction of LBP features from two specific regions of the ROI (core region and ribbon region) and the use of SVM-RFE to select features. Authors used the rotation invariant LBP proposed by Ojala et al. [21]. The experiments were performed using 303 images from DDSM database [5] from which 246 mass ROIs and 2,497 normal tissue ROIs were extracted. The $A_{z}$ of the ROC curve of the proposed approach was in the range of $0.9 \pm 0.02$. Authors also evaluated their approach of LBP feature extraction applying SVM without RFE and the resulting $A_{z}$ was in the range $0.87 \pm 0.03$. The result using SVM was very near the results using SVM-RFE, however the authors did not test the statistical significance of that difference.

Liu et al. [14] proposed a modification in the rotation invariant LBP algorithm [21] in order to use it for mammograms classification (malignant or benign). The proposed modification is the use of the median value of the neighboring pixels as threshold instead of the value of the central pixel. The authors experimented their approach on 309 (142 were benign and 167 were malignant) mammograms from DDSM database [5]. The approach was tested using different window sizes, from $1 \times 1$ to $9 \times 9$ and compared with original LBP [20] and rotation invariant LBP [21]. Except for $1 \times 1$ window size, the Liu et al. [14] approach achieved the best classification rates which ranged from 52.1875 up to 66.1458 .

From all papers discussed in this subsection, the best classification results were obtained by Lladó et al. [16] $(0.94 \pm 0.02)$. Therefore, only that work will be compared with our method in Section 4(Table 5). All the approaches discussed in this subsection used images from DDSM database [5]. Although DDSM has many images available in comparison with MIAS, the authors used only a small amount of them. 


\section{Proposed Approach}

We extracted LBP features from images of the MIAS (Mammographic Image Analysis Society) [17] database by applying a LBP (Local Binary Patterns) operator. The classifier used was the SVM (Support Vector Machine). A brief explanation of the LBP method is given as follows.

Local Binary Patterns (LBP) is a method for texture feature extraction very popular for face detection and recognition approaches. As mentioned in Section 2, texture features are very useful for breast cancer abnormality classification in mammographies and LBP has been used to classify mammograms. However, to the best of our knowledge, our approach is generic enough to be applied in classification of different breast cancer anomalies. What makes LBP features attractive is their robustness to illumination variations. It is also possible to obtain rotation invariance by grouping the extracted features in histograms. In its original version [20], LBP was proposed as a histogram of binary values. Those binary values are computed if a neighbor value is higher than the central value 1 is assigned to that position, otherwise 0 is assigned. The two main user defined parameters in the LBP method are $P$ (number of neighbors) and R (radius of comparison). The procedure is illustrated in Figure 1 It is possible to obtain rotation invariance by grouping the extracted features in histograms.

\begin{tabular}{|l|l|l|}
\hline 60 & 70 & 40 \\
\hline 30 & 50 & 20 \\
\hline 90 & 40 & 70 \\
\hline
\end{tabular}
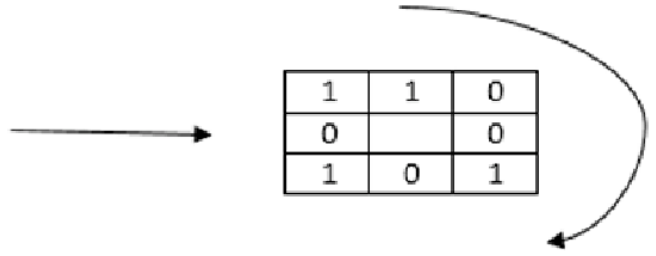

Figura 1. LBP operator in action.

According to Figure 1 the binary code obtained from that image region is: $(01100101)_{2}=$ $(101)_{10}$. In Table 1 the parameter variations used in the experiments performed for this paper are presented.

In our experiments, the initial vector size obtained by concatenation of all extracted LBP features was 66,080 (using $P=\{4,8,16\}$ and $R=\{1,2\}$, as in Table 11, which is excessively high for a SVM classifier due to the required processing time. To reduce the vector size, we decided to use only the following values of $P$ and $R$ : $(\mathrm{P}=4, \mathrm{R}=1),(\mathrm{P}=4, \mathrm{R}=2)$, $(\mathrm{P}=8, \mathrm{R}=1)$, and $(\mathrm{P}=8, \mathrm{R}=2)$. The resulting number of features was 544 , still too high. Finally, the number of histogram bins was reduced by half (using only: $(\mathrm{P}=4, \mathrm{R}=1)$ and $(\mathrm{P}=8, \mathrm{R}=1)$ ), yielding in an acceptable total of 272 features, which were used in the experiments described next. 
Tabela 1. Tested LBP Parameters $P$ and $R$ and the number of extracted features $N$.

\begin{tabular}{lll}
\hline $\mathrm{P}$ & $\mathrm{R}$ & $\mathrm{N}$ \\
\hline 4 & 1 & $2^{4}=16$ \\
& 2 & \\
8 & 1 & $2^{8}=256$ \\
& 2 & \\
16 & 2 & $2^{8}=65536$ \\
\hline
\end{tabular}

\section{Results and Discussion}

This section presents the experimental results obtained by our approach for detection of cancer candidate regions in breast mammographies. Initially, the experimental setup (used images, classifiers, etc) is presented, followed by results and discussions.

\subsection{Experimental Setup}

All experiments were performed using LBP features and the SVM implementation called libsvm [2, 10], which is publicly available. This classifier was chosen because it is very robust and known as one of the best off-the-shelf classifiers available. The SVM classifiers used in this research were trained with RBF (Radial Basis Function) kernel, and parameters obtained by a 10 -fold cross validation, varying the values of cost and gamma.

For the experiments we used images from MIAS database (Mammographic Image Analysis Society) [17] This database consists of 322 images grouped in 6 breast cancer classes: CALC (calcification), CIRC (well-defined masses), SPIC (spiculated masses), MISC (other types of masses, ill-defined), ARCH (architectural distortion), and ASYM (asymmetry). Each image of the database has resolution of $1024 \times 1024$, and 8-bit depth. For all images containing cancer regions there is the annotation (coordinates of a circular region and the corresponding radius) of the region made by a physician. Figure 2 shows two samples of MIAS images.

Due to the small size of the MIAS base the set of image crops for positive samples had to be synthetically increased for our experiments. The procedure utilized for that consisted of successively translating the center of the cancer region by 1 pixel above, left, right, down, and combinations. This resulted in increasing the number of samples by a factor of 7 for each class cancer type, as shown in Table 2 In Figure 3, the procedure of cropping new samples is illustrated. The use of synthetic images for supervised classification has been utilized

$5 \longdiv { \text { http://peipa.essex.ac.uk/info/mias.html } }$ 

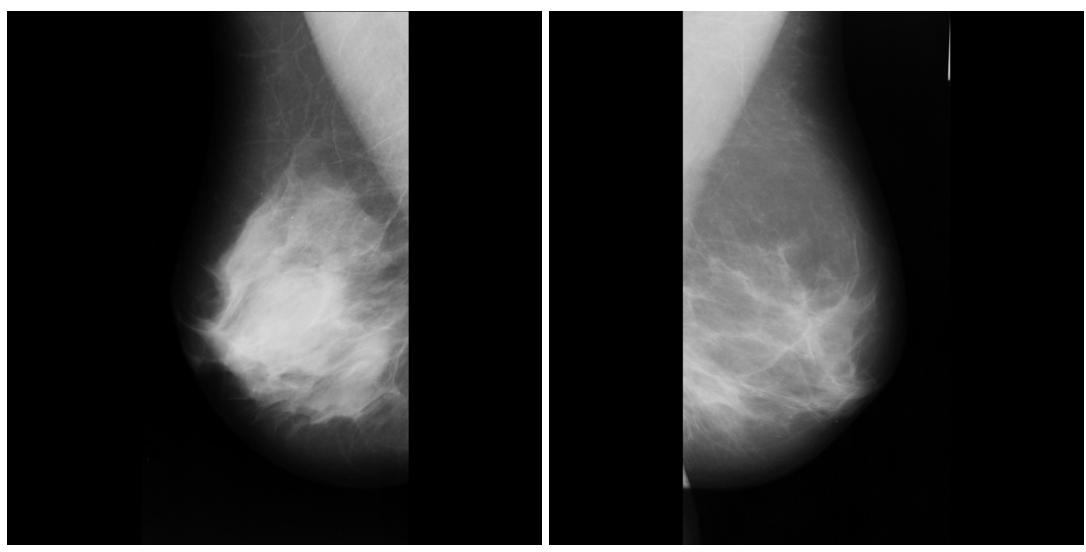

Figura 2. Two samples of images obtained from the MIAS database.

for solving computer vision problems such as handwritten character recognition [19]. The horizontal and vertical shifting procedure we utilized for samples generation is based on the work by Varga and Bunke [27].

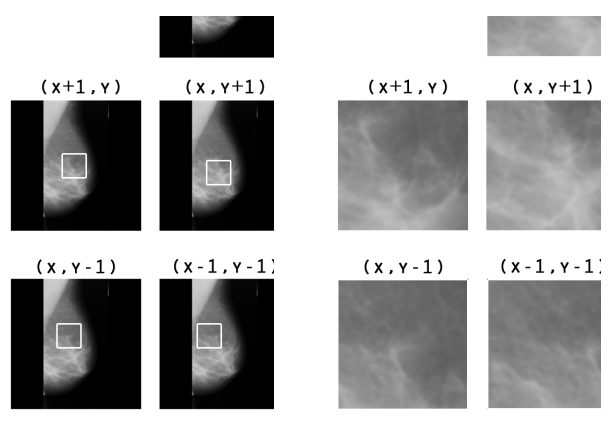

Figura 3. Illustration of Crops.

The NORMAL class was obtained by cropping regions that do not contain cancer (according to the database description). All crops used in the experiments had resolution of $40 \times 40$ pixels. The amount of images used for training and testing correspond, respectively, to $60 \%$ and $40 \%$ of the total available, except for the NORMAL class for which 500 samples 
Tabela 2. Quantity of images and regions of interest (ROI) cropped from MIAS images.

\begin{tabular}{lll}
\hline Cancer Type & Quantity of Images & Quantity of ROI \\
\hline CALC (Calcification) & 25 & 175 \\
CIRC (Well-defined/circumscribed masses) & 20 & 140 \\
SPIC (Spiculated masses) & 19 & 133 \\
MISC (Other, ill-defined masses) & 15 & 105 \\
ARCH (Architectural distortion) & 19 & 133 \\
ASYM (Asymmetry) & 15 & 105 \\
NORMAL & - & 50694 \\
\hline
\end{tabular}

were used for both training and testing. Table 3 shows the amounts of used images for all classes. The reasons for using a higher amount of images for NORMAL than for other classes are:

- NORMAL is the negative class, occurring with higher probability in mammographies than any other class corresponding to a cancer region;

- Possibilities of different NORMAL class image regions are practically infinite, therefore it is necessary to train and test that class using a as large as possible amount of images;

- The number 500 was chosen as an approximation for 5 times the highest quantity of images available for training the other classes.

In other well established pattern recognition areas such as face detection it is common to use hundred of thousands or even millions of negative samples for training [22]. However, as the SVM classifier is affected by the curse of dimensionality, it is not possible to use thousands or millions of NORMAL class images without resorting to some type of parallelization approach. As speed performance was not the focus of the present work, we decided to use only 1,000 NORMAL class images (500 for training and 500 for testing).

The approach for extracting NORMAL regions may produce black pixels only, extracted from background areas as shown in Figure 2. In order to reduce such occurrences, a selection procedure was applied, resulting in two sets of NORMAL samples: non-filtered, and filtered. Filtered samples are those that have over $25 \%$ of their pixels different from zero, i.e., more than $75 \%$ of the pixels in the cropped region are not black.

After setting up the image crops for training and testing, a 10-fold cross validation procedure was performed using the training set to choose the best parameters for training SVM with RBF kernel. 
Tabela 3. Quantity of ROIs used for training and testing SVM classifiers.

\begin{tabular}{lll}
\hline Class & Training & Testing \\
\hline Class 0 (NORMAL) & 500 & 500 \\
Class 1 (CIRC) & 84 & 55 \\
Class 2 (CALC) & 105 & 69 \\
Class 3 (SPIC) & 80 & 53 \\
Class 4 (MISC) & 63 & 41 \\
Class 5 (ARCH) & 79 & 52 \\
Class 6 (ASYM) & 62 & 40 \\
\hline
\end{tabular}

Next, results for the two types of NORMAL images are presented.

\subsection{Experimental Results}

Two types of experiments were performed, one using the filtered and the other using non-filtered NORMAL samples. Other than NORMAL, there are 6 breast cancer classes: CIRC, CALC, SPIC, MISC, ARCH, and ASYM.

Tabela 4. Values of area under the ROC curve (AUC) for experiments using our proposed approach.

\begin{tabular}{lllll}
\hline \multicolumn{1}{c}{ Class } & \multicolumn{2}{c}{ Class Value } & \multicolumn{2}{c}{ Not-Cancer Value } \\
& Non-Filtered & Filtered & Non-Filtered & Filtered \\
\hline Class 1 (CIRC) & 0.9743 & 0.9568 & 0.9743 & 0.9568 \\
Class 2 (CALC) & 0.9898 & 0.9993 & 0.9898 & 0.9993 \\
Class 3 (SPIC) & 0.9906 & 0.9807 & 0.9906 & 0.9807 \\
Class 4 (MISC) & 0.9846 & 0.9947 & 0.9846 & 0.9947 \\
Class 5 (ARCH) & 0.9859 & 0.9735 & 0.9859 & 0.9735 \\
Class 6 (ASYM) & 0.9923 & 0.9915 & 0.9924 & 0.9916 \\
\hline
\end{tabular}

The AUC results presented in Table 4 for our method are higher than those of Lan et al. [13] who obtained an 0.86 for masses detection in mammographies using DDSM images (see Section 2). Our approach was capable of classifying any of the six different breast cancer types, including masses, and obtained AUC higher than 0.95 in all cases. A possible explanation for our results being higher is that SVMs are more robust classifiers than the classifiers used by Lan et al. [13]: LR (Logistic Regression) and KNN (K-Nearest Neighbors).

The AUC result obtained by our approach for Class 2 (CALC) was 0.999 , higher than 
the 0.963 result obtained by Zhang and Xie [28] using DDSM images and higher than the result 0.876 obtained by Nguyen et al. [18] using the MIAS database. Zhang and Xie [28] used only 65 cancer images for training and 65 for testing, while we used 105 calcification images for training and 69 for testing. This difference between training set sizes may explain the higher AUC achieved by our LBP-SVM method. Nguyen et al [18] used the same image database used in this paper, the MIAS database. However, they used only 12 features for training the neural network. In our approach (LBP+SVM), 272 texture features were used, which explains our results being better than those presented by Nguyen et al. [18].

In Figure 4 the ROC curves are presented for each breast cancer class. It may be seen that for all classes, the classifiers start with high true positive rates and $0 \%$ of false positive rate. In 4 cases (CIRC, CALC, MISC and ARCH), the initial true positive rate is equal or higher than $90 \%$. In the other 2 cases (SPIC and ASYM), the starting true positive rate is higher than $80 \%$, and very near to $90 \%$, respectively.

In Table 5 we show results from reviewed works as well as the results obtained by our approach. Except for Hussain et al.[11], our method obtained AUC higher than all other approaches. However, it should be observed the small amount of samples used by Hussain et al. [11] for training and testing, 89 regions of interest acquired from MIAS for masses detection only, which compromises comparison. Another important point is that, differently from all other methods found, our approach was tested for all six different breast cancer types, and proved to be generic enough to obtain high classification rates in all cases. For simplifying comparison only results for calcification and circular masses (classes 1 and 2) are presented in Table 5 . This simplification is in agreement with the majority of other published works, which only evaluate those two classes of abnormalities in mammographies.

\section{Conclusions}

In this paper, we propose a breast cancer classification method for mammographies using texture features represented by local binary patterns (LBP) and a SVM classifier. Our method has been tested for six different types of cancer indicating abnormalities: CALC, CIRC, SPIC, MISC, ARCH, ASYM. A public image database was used for training and testing. For all experiments, ROC curves were obtained and the AUC was computed. In all cases the results were higher than 0.8. Results from five other published approaches were discussed in order to have an idea about the quality of our approach results. To trace a comparison among our results and the mentioned works is practically impossible due to absence of a benchmark test dataset shared by researchers in this area. Although MIAS and DDSM are well used each research reviewed used different amounts of images or resized the images for different pixels resolutions. A plus of our approach is that, contrarily to other methods, it was evaluated on all six considered breast cancer types and presented high performance. 


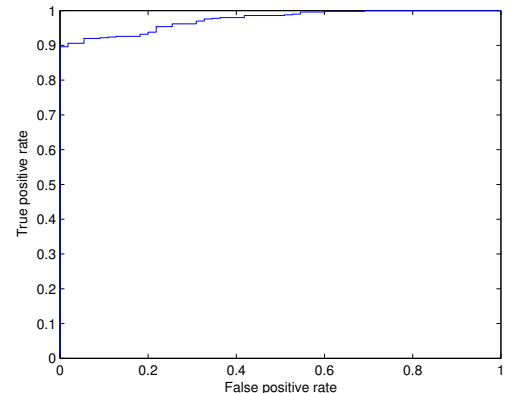

(a) ROC curve for classification of CIRC.

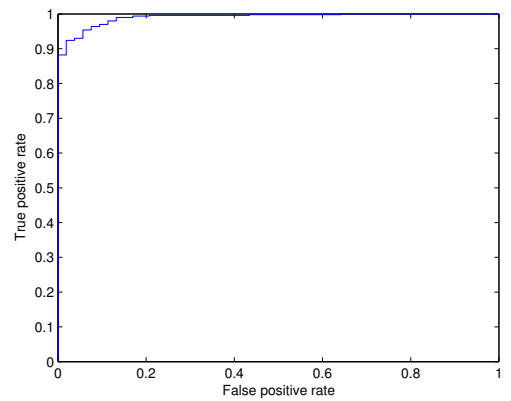

(c) ROC curve for classification of SPIC.

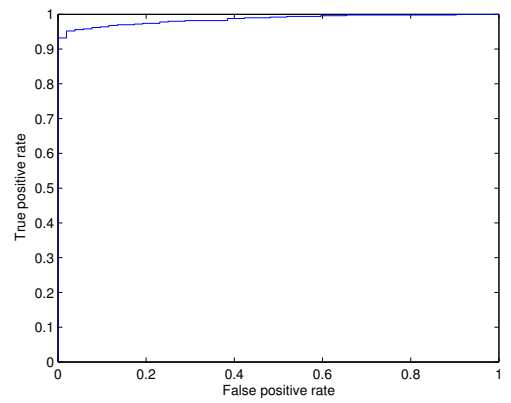

(e) ROC curve for classification of ARCH.

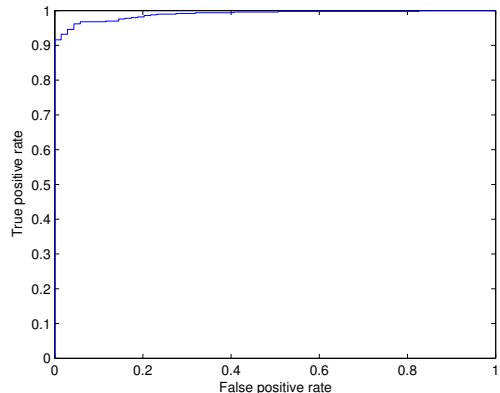

(b) ROC curve for classification of CALC.

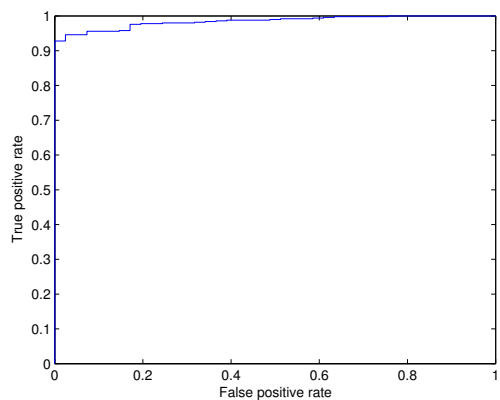

(d) ROC curve for classification of MISC.

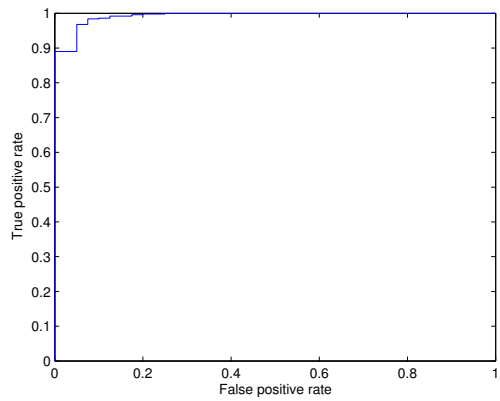

(f) ROC curve for classification of ASYM.

Figura 4. ROC curves for classification of six different types of breast cancer versus NORMAL breast regions. 
Tabela 5. Results for breast cancer detection in mammographies.

\begin{tabular}{|c|c|c|c|c|c|}
\hline Authors & $\begin{array}{l}\text { Extracted } \\
\text { Features }\end{array}$ & Classifier & $\begin{array}{l}\text { Cancer } \\
\text { Type }\end{array}$ & $\begin{array}{c}\text { Image } \\
\text { Database }\end{array}$ & AUC \\
\hline Lan et al.[13] & $\begin{array}{c}\text { gray levels } \\
\text { texture } \\
\text { shape } \\
\text { edges }\end{array}$ & LR and KNN & Masses & DDSM & 0.86 \\
\hline Nguyen et al.[18] & GLCM and Haralick & Neural Networks & Masses & MIAS & 0.88 \\
\hline Zhang and Xie 28 & $\begin{array}{c}\text { histograms } \\
\text { Chebyshev } \\
\text { Zernike }\end{array}$ & TWSVM & Microcalcifications & DDSM & 0.96 \\
\hline Hussain et al. 11] & $\begin{array}{l}\text { PCA and LDA } \\
\text { applied to } \\
\text { Gabor features }\end{array}$ & SELwSVM & Masses & MIAS & 1 \\
\hline Llado et al. [16] & region based LBP & SVM-RFE & Masses & DDSM & 0.94 \\
\hline $\begin{array}{l}\text { Proposed } \\
\text { Approach }\end{array}$ & LBP & SVM & $\begin{array}{l}\text { CIRC, CALC, SPIC, } \\
\text { MISC, ARCH, ASYM }\end{array}$ & MIAS & $\begin{array}{l}0.97 \\
0.99\end{array}$ \\
\hline
\end{tabular}

As future work, we intend to perform tests using rotation invariant LBP techniques, as well as other image databases. Another possibility of future work is evaluation of other classifiers for breast cancer classification and applying the proposed approach for cancer region detection in mammographic images.

\section{References}

[1] J. Anitha and J. Peter. A wavelet based morphological mass detection and classification in mammograms. In Machine Vision and Image Processing (MVIP), 2012 International Conference on, pages 25-28, 2012.

[2] C.-C. Chang and C.-J. Lin. Libsvm - a library for support vector machines. http://www.csie.ntu.edu.tw/ cjlin/libsvm/.

[3] J. Y. Choi, D. H. Kim, and Y. M. Ro. Combining multiresolution local binary pattern texture analysis and variable selection strategy applied to computer-aided detection of breast cancer masses on mammograms. In International Conference on Biomedical and Health Informatics, pages 495-498, 2012.

[4] J. Davis and M. Goadrich. The relationship between precision-recall and roc curves. In Proceedings of the 23rd International Conference on Machine Learning, pages 233240, 2006.

[5] DDSM. Digital database for screening mammography. http://marathon.csee.usf.edu/Mammography/Database.html. 
[6] J. Dinnes, S. Moss, J. Melia, R. Blanks, F. Song, and J. Kleijnen. Effectiveness and costeffectiveness of double reading of mammograms in breast cancer screening: findings of a systematic review. The Breast, 10(6):455-463, 2001.

[7] F. J. Gilbert, S. M. Astley, M. A. McGee, M. G. C. Gillian, C. R. M. Boggis, P. M. Griffiths, and S. W. Duffy. Single reading with computer-aided detection and double reading of screening mammograms in the united kingdom national breast screening program. Radiology, 241(1):47-53, 2006.

[8] M. Gromet. Comparison of computer-aided detection to double reading of screening mammograms: Review of 231,221 mammograms. American Journal of Roentgenology, 190(4):854-859, 2008.

[9] I. Guyon, J. Weston, S. Barnhill, and V. Vapnik. Gene selection for cancer classification using support vector machines. Machine Learning, 46:389-422, 2002.

[10] C.-W. Hsu, C.-C. Chang, and C.-J. Lin. A practical guide to support vector classification. http://www.csie.ntu.edu.tw/ cjlin/papers/guide/guide.pdf, 2010.

[11] M. Hussain, S. Khan, G. Muhammad, and G. Bebis. A comparison of different gabor features for mass classification in mammography. In Signal Image Technology and Internet Based Systems (SITIS), 2012 Eighth International Conference on, pages 142148, 2012.

[12] INCA. Instituto nacional de câncer. http://www.inca.gov.br.

[13] Y. Lan, H. Ren, and J. Wan. A hybrid classifier for mammography cad. In Computational and Information Sciences (ICCIS), 2012 Fourth International Conference on, pages 309-312, 2012.

[14] J. Liu, X. Liu, J. Chen, and J. Tang. Improved local binary patterns for classification of masses using mammography. In IEEE International Conference on Systems, Man, and Cybernetics, pages 2692-2695, 2011.

[15] X. Liu, X. Xu, J. Liu, and Z. Feng. A new automatic method for mass detection in mammography with false positives reduction by supported vector machine. In International Conference on Biomedical Engineering and Informatics, pages 33-37, 2011.

[16] X. Lladó, A. Oliver, J. Freixenet, R. Martí, and J. Martí. A textural approach for mass false positive reduction in mammography. Computerized Medical Imaging and Graphics, 33:415-422, 2009.

[17] MIAS. The mini-mias database of mammograms. http://peipa.essex.ac.uk/info/mias.html. 
[18] V. Nguyen, D. Nguyen, H. Nguyen, D. Bui, and T. Nguyen. Automatic identification of massive lesions in digitalized mammograms. In Communications and Electronics (ICCE), 2012 Fourth International Conference on, pages 313-317, 2012.

[19] J. Nonnemaker and H. S. Baird. Using synthetic data safely in classification. In SPIE Document Recognition and Retrieval, pages 1-11, 2009.

[20] T. Ojala, M. Pietikäinen, and D. Harwood. A comparative study of texture measures with classification based on featured distributions. Pattern Recognition, 29(1):51-59, 1996.

[21] T. Ojala, M. Pietikäinen, and T. Mäenpää. Multiresolution gray-scale and rotation invariant texture classification with local binary patterns. IEEE Transactions on Pattern Analysis and Machine Intelligence, 24:971-987, 2002.

[22] E. T. Pereira, H. M. Gomes, and J. M. de Carvalho. An approach for multi-pose face detection exploring invariance by training. In J. F. M. Trinidad, J. A. C. Ochoa, J. A. O. Lopez, J. Salas-Rodríguez, and C. Y. Suen, editors, Pattern Recognition, Lecture Notes in Computer Science, volume 8495, pages 182-191. Springer International Publishing, Switzerland, 2014.

[23] E. T. Pereira, H. M. Gomes, E. S. Moura, J. M. de Carvalho, and T. Zhang. Investigation of local and global features for face detection. In IEEE Symposium on Computational Intelligence for Multimedia, Signal and Vision Processing (CIMSIVP), pages 114-121, 2011.

[24] M. Pietkäinen, A. Haddid, G. Zhao, and T. Ahonen. Computer Vision Using Local Binary Patterns. Springer, 2011.

[25] R. M. Rangayyan, F. J. Ayres, and J. L. Desautels. A review of computer-aided diagnosis of breast cancer: Toward the detection of subtle signs. Journal of the Franklin Institute, 344(3 - 4):312 - 348, 2007.

[26] R. Smith. Netters Obstetrics and Gynecology. Saunders Elsevier, 2008.

[27] T. Varga and H. Bunke. Generation of synthetic training data for an hmm-based handwriting recognition system. In International Conference on Document Analysis and Recognition, pages 618-622, 2003.

[28] X. Zhang and H. Xie. A new approach for clustered microcalcifications detection. In Information Processing, 2009. APCIP 2009. Asia-Pacific Conference on, volume 2, pages 322-325, 2009. 